\title{
BMJ Open Clinical teaching unit design: a realist systematic review protocol of evidence- based practices for clinical education and health service delivery
}

\author{
Brandon Tang (D) , ${ }^{1}$ Ryan Sandarage (D) , ${ }^{1}$ Katrina Dutkiewicz, ${ }^{1}$ Stephan Saad, ${ }^{1}$ \\ Jocelyn Chai, ${ }^{1}$ Kristin Dawson, ${ }^{1}$ Vanessa Kitchin, ${ }^{1}$ lain McCormick, ${ }^{1}$ \\ Barry Kassen ${ }^{1,2}$
}

To cite: Tang B, Sandarage $\mathrm{R}$, Dutkiewicz K, et al. Clinical teaching unit design: a realist systematic review protocol of evidence-based practices for clinical education and health service delivery. BMJ Open 2020;10:e034370. doi:10.1136/ bmjopen-2019-034370

- Prepublication history and additional material for this paper are available online. To view these files, please visit the journal online (http://dx.doi org/10.1136/bmjopen-2019034370).

BT and RS contributed equally.

Received 20 September 2019

Revised 08 January 2020

Accepted 06 February 2020

\section{Check for updates}

(c) Author(s) (or their employer(s)) 2020. Re-use permitted under CC BY-NC. No commercial re-use. See rights and permissions. Published by BMJ.

${ }^{1}$ Department of Medicine, The University of British Columbia, Vancouver, British Columbia, Canada

${ }^{2}$ St. Paul's Hospital, Vancouver, British Columbia, Canada

Correspondence to

Dr Brandon Tang;

bran.tang@mail.utoronto.ca

\section{ABSTRACT}

Introduction The clinical teaching unit (CTU) has emerged as a near-ubiquitous model of clinical education across Canadian and international medical schools since it was first proposed over 50 years ago. However, while healthcare has changed dramatically over this period, the CTU model has remained largely unchanged. We thus aimed to systematically review principles of CTU design that contribute to improved outcomes in clinical education and health service delivery.

Methods and analysis We will perform a realist systematic review in accordance with the Realist And Meta-narrative Evidence Syntheses: Evolving Standards (RAMESES) II protocol for realist reviews. Databases, including MEDLINE, Embase, Cochrane Database of Systematic Reviews and Cumulative Index of Nursing and Allied Health Literature (CINAHL), were searched to find primary research articles published from 1993 to 2019 involving CTUs or other teaching wards, and outcomes related to either trainee education or health service delivery. Two reviewers will independently screen studies in a two-stage process. Retrieved titles and/or abstracts of studies will be screened in the first stage, with full texts reviewed in the second stage. Selected articles meeting inclusion criteria will undergo data abstraction using a standardised, pre-piloted form for assessment of study quality and knowledge synthesis.

Ethics and dissemination This review will generate higher quality evidence on the design of CTUs as a model for both clinical education and health service delivery. In addition, further knowledge translation efforts may be necessary to ensure that known best practices in CTU design become common practice.

\section{INTRODUCTION}

The clinical teaching unit (CTU) model of clinical education was first approved by the Association of Canadian Medical Colleges (ACMC) in 1962. ${ }^{1}$ The approach aimed to organise teaching hospitals to accommodate a growing number of medical trainees in context of limited clinical teachers. Later, the proposed core principles of the CTU
Strengths and limitations of this study

- This will be the first comprehensive review of clinical teaching unit (CTU) design for undergraduate and postgraduate medical education, and may help establish guidelines for developing CTUs.

- A realist approach to systematic review is designed to understand 'what works, in what circumstances and how' for complex interventions such as CTU, and is therefore likely to generate outcomes relevant to policy change.

- The search strategy has been developed by academic and clinical research team members (including a medical sciences librarian) with content and methodological expertise.

- Given that most retrieved studies involve qualitative approaches, the strength of recommendations that emerge from this review may be limited.

- While this systematic review will include all article types and methodologies, there is a limitation in that the articles included are limited to English language.

included: (1) residency training providing an educational experience which simulates realworld practice wherever possible; (2) creating an exemplary standard of care for both the patient and broader community and (3) fostering a favourable environment for scientific endeavour. ${ }^{2}$

A subsequent formal definition of CTU published by the ACMC in $1966^{3}$ explicitly embraced the concepts posited by previous studies. $^{2}$ Additionally, it defined logistical aspects of CTU, for example, recognising that CTU designates certain clinical wards as appropriate for undergraduate and postgraduate medical education, under the auspices of a university-associated faculty of medicine. The ACMC also stated that CTU staff should be jointly appointed by both the hospital and university. Finally, it suggested that patient care is a shared responsibility among a 
clinical team including a staff physician and trainees at various levels, who assume graded responsibility reflective of their level of training.

\section{CTU design}

While many of the original principles of CTU still hold true today, the broader health system landscape has changed dramatically, including changing population and disease demographics, novel medical technologies, shifts in healthcare delivery and novel professional roles for healthcare providers. ${ }^{4}$ Hence, while the CTU model has remained largely unchanged over the past 50 years, it is unclear whether it reflects the demands of today's healthcare environment.

Our initial review of the literature suggests that evidence-based design of modern CTUs is limited. Some common practices such as structured interdisciplinary rounds and hospitalist-run short-stay units appear to have some degree of proven benefit. ${ }^{56}$ However, modern practices in CTU design are largely based on clinical intuition and experience, as opposed to empirical evidence. The proposed systematic review aims to identify principles of CTU design that contribute to improved outcomes in clinical education and health service delivery. We are hopeful that identifying evidence-based principles of CTU design will help inform redesign of the modern CTU, in accordance with the reality of healthcare in the 21st century.

Our project will lay the foundations for: (1) current resource allocation and staffing for CTUs, (2) future intervention-based studies on principles of CTU design and (3) alternative models of clinical education. This project, therefore, holds considerable potential for both immediate educational and health system relevance.

\section{METHODS AND ANALYSIS \\ Design}

The proposed study will use a methodologically rigorous realist approach to systematic review in accordance with the Realist And Meta-narrative Evidence Syntheses: Evolving Standards (RAMESES) II publication standards for realist reviews. ${ }^{78}$ Our study began on 1 April 2019 with an anticipated end date of 1 December 2020. A comprehensive literature search strategy has been developed and executed with the assistance of a medical librarian. Appropriate inclusion and exclusion criteria have been developed. Currently, retrieved articles are undergoing a two-step screening process, in accordance with Preferred Reporting Items for Systematic Reviews and Meta-Analyses reporting guidelines, to select full-text articles for inclusion in the study, presented in online supplementary file 1. A data collection spreadsheet will be designed to extract both a priori and data-driven themes of CTU design from selected articles. Findings will be communicated via both qualitative and quantitative synthesis in accordance with realist review approaches.

Our review was limited to include studies published in 1993 or later, which would be more likely to reflect aspects of CTU relevant to today's healthcare environment. This cut-off year was selected given the publication of a landmark opinion article in 1993 which called for reform and modernisation of the CTU. ${ }^{1}$

\section{Study objectives}

- Primary question:

- Which principles of CTU design contribute to improved outcomes in clinical education and health service delivery?

- Secondary questions:

- In what contexts (country, clinical specialty and so on) has the design of CTUs been examined?

- Which principles of CTU design have the highest quality supporting evidence?

- Which interventions in CTU design have the largest impact on clinical education and health service delivery?

- What gaps exist in the CTU design literature that suggest areas for future study?

\section{Eligibility criteria}

Inclusion criteria

This review will include the following studies:

- English language studies.

- Primary research studies (including but not limited to observational, descriptive and survey studies).

- Studies based in an inpatient CTU (or any teaching unit) that involves either resident physicians and/or medical students.

- Studies with outcomes related to trainee education and/or health service delivery.

\section{Exclusion criteria}

- Non-English studies.

- Not a primary research study, including systematic reviews, meta-analyses, conference abstracts, and editorial/opinion articles.

- Study is based in a non-CTU setting, or setting that does not involve the education of either resident physicians or medical students.

- Outcomes do not relate to either trainee education or health service delivery.

- Publication date 1992 or earlier (inclusive).

We did not limit our inclusion criteria to interventionbased studies, after preliminary literature searches suggested that many studies with outcomes relevant to CTU design were qualitative or observational, but without a discrete intervention.

\section{Search strategy to identify relevant studies}

We searched Ovid MEDLINE (1946 to present, in-process and other non-indexed citations), Ovid Embase (1974 to 21 May 2019), Ovid EBM reviews-Cochrane Database of Systematic Reviews (2005 to 21 May 2019), Ovid EBM reviews-Cochrane Central Register of Controlled Trials (May 2019), EBSCO Cumulative Index of Nursing and Allied Health Literature (CINAHL) (1982 to present), EBSCO PsycINFO (1806 to present), EBSCO ERIC (1969 
to present), ProQuest Dissertations and Theses Global (1861 to present) and UBC's CiRcle (institutional repository of dissertations and theses), to identify articles addressing the subjects of teaching units and teaching teams in academic medical centres. The search strategies were translated using each database platform's command language, controlled vocabulary and appropriate search fields. Medical subject heading terms, Emtree terms, CINAHL subject headings terms, American Psychological Association thesaurus terms and text words were used for the search concepts of 'clinical teaching unit', 'teaching rounds', 'academic medical centres' and 'residents/medical students'. Searches were limited to articles published from 1993 onwards. A sample search strategy can be found in the online supplementary file 2. Restrictions according to status of publication (eg, in review, accepted, in press) or specific types of records (eg, commentaries, letters, editorials) were not applied. We limited our study to English language articles given the limited resources at our institution to appropriately search, screen and analyse studies in languages other than English.

\section{Selection of studies for inclusion}

Two reviewers will independently screen studies in a twostage process. In the first stage, titles and/or abstracts of studies retrieved using the search strategy and those from additional sources will be independently screened by two review authors using Covidence software, to identify studies that potentially meet inclusion criteria. In the second stage, the full text of these potentially eligible studies will be retrieved and independently assessed for eligibility by two review authors. Any disagreement over the eligibility of studies will be resolved through discussion with a third reviewer.

\section{Data extraction and management}

A standardised, pre-piloted form will be used to extract data from included studies for assessment of study quality and evidence synthesis. Extracted information may include: study purpose; study setting; study population; participant demographics and baseline characteristics (both patients and clinical trainees); details of the intervention and control conditions (if applicable); study design; recruitment and study completion rates; outcomes and times of measurement (related to clinical education and/or health service delivery); categorisation of the study according to the Star Model (strategy, structure, human resources, incentives, information and decision support, and culture $)^{9}$; suggested mechanisms of intervention action; information for assessment of the risk of bias. However, the data abstraction form will be iteratively refined throughout the data abstraction process based on emergent outcomes and themes from included articles. Two review authors will extract data independently, and discrepancies will be identified and resolved through discussion (with a third author where necessary). Missing data will be requested from original study authors.

\section{Data synthesis and analysis}

We intend to employ a realist approach to knowledge synthesis. Realist research uses mixed methods (qualitative and quantitative) data to generate contextmechanism-outcome (CMO) hypotheses regarding the intervention of interest. In other words, it aims to understand 'what works for whom, in what circumstances, in what respects and how' for complex interventions such as CTU. ${ }^{7}$ Based on an analysis of knowledge synthesis strategies by Kastner et al, ${ }^{10}$ a realist review approach is appropriate for our study for multiple reasons:

- Purpose: our study aims to generate theory regarding why and how certain principles of CTU design are effective, as well as identify gaps in the CTU design literature.

- Outputs: given the wide range of contexts in which CTU occurs and has been studied, a realist approach is ideal given that it aims to generate highly contextualised CMO statements. By considering what works in CTU design for whom, and in what circumstances, the results of our study will be highly relevant to our intended audience of clinician teachers, educators and administrators.

- Applicability: our study involves the evaluation and/ or assessment of a complex programme (CTU).

If the necessary data are available, subgroup analyses will be done for patients/clinical trainees by paediatric or adult inpatient settings, and/or by the service for which they are receiving care (internal medicine, surgery, psychiatry and so on). We will aim to categorise emergent principles of CTU design according to the Star Model, an evidence-based management tool used to guide health system design. ${ }^{911}$ This framework examines macro-systems (such as CTU) according to their interdependent subsystems (strategy, structure and so on), and may help facilitate policy reform and communication by segmenting CTU into conceptual components meaningful to educators and administrators alike. Additionally, we believe that applying a framework intended for health system design may facilitate both systems thinking and process improvement in the CTU context. Risk of bias assessment will be performed for all included studies using validated tools such as the Newcastle-Ottawa Scale for qualitative studies or Grading of Recommendations, Assessment, Development and Evaluations (GRADE) system for quantitative studies.

\section{Potential amendments}

We anticipate that there will be diverse context for included studies, such as clinical specialty, country and study designs. Given that our realist review and the emergent CMO hypotheses can only account for limited contextual variability, our published analysis may focus on a subset of retrieved articles, such as only those from internal medicine CTUs. This will increase the relevance and generalisability of our findings to the end users within a particular field, such as educators and administrators within internal medicine. 
We do not plan to make any additional changes to this protocol. However, if substantial changes occur during the review process, they will be reported in the published results.

\section{Patient and public involvement}

No patients involved.

\section{Ethics and dissemination}

This paper presents the protocol for a systematic review of CTUs for undergraduate and postgraduate medical education. This review will advance knowledge on CTUs, generating higher quality evidence on their design. Through dissemination in publications and relevant conferences, the results may guide future design of CTUs to optimise medical trainee education.

Contributors BT conceptualised and directed the project. BT, KD, SS, IM and $\mathrm{BK}$ designed the project. VK and BT developed and executed the search strategy. BT and RS developed the study protocol. BT, KD, SS, RS, JC and KD will screen prospective studies, extract and analyze data and prepare the final manuscript for publication.

Funding This work was supported by University of British Columbia Division of General Internal Medicine.

Competing interests None declared.

Patient consent for publication Not required.

Provenance and peer review Not commissioned; externally peer reviewed.

Open access This is an open access article distributed in accordance with the Creative Commons Attribution Non Commercial (CC BY-NC 4.0) license, which permits others to distribute, remix, adapt, build upon this work non-commercially, and license their derivative works on different terms, provided the original work is properly cited, appropriate credit is given, any changes made indicated, and the use is non-commercial. See: http://creativecommons.org/licenses/by-nc/4.0/.
ORCID iDs

Brandon Tang http://orcid.org/0000-0002-1969-7595

Ryan Sandarage http://orcid.org/0000-0002-7998-5505

\section{REFERENCES}

1 Maudsley RF. The clinical teaching unit in transition. CMAJ 1993;148:1564-6.

2 Evans JR, Chute AL, Morley TP. The clinical teaching unit as an effective organization for the education of residents under changing medical socio-economic circumstances. I. objectives and organization of the clinical teaching unit: a white paper. Can Med Assoc J 1966;95:720-7.

3 Schrewe B, Pratt DD, McKellin WH. Adapting the forms of yesterday to the functions of today and the needs of tomorrow: a genealogical case study of clinical teaching units in Canada. Adv in Health Sci Educ 2016;21:475-99.

4 Towle A. Continuing medical education: changes in health care and continuing medical education for the 21 st century. BMJ 1998;316:301-4.

5 O'Leary KJ, Buck R, Fligiel HM, et al. Structured interdisciplinary rounds in a medical teaching unit: improving patient safety. Arch Intern Med 2011;171:678-84.

6 Abenhaim HA, Kahn SR, Raffoul J, et al. Program description: a hospitalist-run, medical short-stay unit in a teaching hospital. C Can Med Assoc J 2000;163:1477-80.

7 Pawson R, Greenhalgh T, Harvey G, et al. Realist review--a new method of systematic review designed for complex policy interventions. J Health Serv Res Policy 2005;10 Suppl 1:21-34.

8 Wong G, Westhorp G, Manzano A, et al. RAMESES II reporting standards for realist evaluations. BMC Med 2016;14:96.

9 Golden BR, Martin RL. Aligning the stars: Using systems thinking to (re)design Canadian healthcare. Healthc Q 2004;7:34-42.

10 Kastner M, Antony J, Soobiah C, et al. Conceptual recommendations for selecting the most appropriate knowledge synthesis method to answer research questions related to complex evidence. $J$ Clin Epidemiol 2016;73:43-9.

11 Galbraith JR. Designing organizations: an executive guide to strategy, structure, and process. San Francisco: Jossey-Bass Publishing, 2001. 